\title{
Developments and Changes Required to Convert Construction Machines to Fully Autonomous Robot During Robot Adoption in the Construction Industry
}

\author{
Dr. Sachin Jain \\ National Institute of Construction Management and Research, Pune,
}

\begin{abstract}
Robots use in construction has many benefits; however a fully autonomous robot cannot be directly adopted in construction, because of complex integration. Robot Autonomy Spectrum helps in such adoption as it considers construction machine on one side and fully autonomous robot on the other side. Hence, in this paper, we try to find various developments which are needed in construction machinery so that it becomes a fully autonomous robot. This paper helps robot developers to develop various versions of the robot so that robot can be adopted in the construction in a smooth way.
\end{abstract}

Keyword: Robot autonomy Spectrum, construction industry, cloud robot

\section{Introduction}

There is a growth in the construction industry worldwide; also as construction has many characteristics like large labor force, stagnancy in technology, minimum R\&D and safety problems which can be solved by the adoption of robots as these robots helps in improvement in productivity, safety, quality works. There is a huge potential for robotics, as robots can help in all stages of the construction life cycle. If the robot is required to be used in the construction then fully autonomous robot will be introduced in stages as per Robot autonomy Spectrum(Sachin and Suman, 2016). Also, as per (Mohr, et al., 2009) during high technology products initially product platform has common architecture which is based on a single design along with technology; and new product platforms known as the next generation will improve performance benefits. This platform and derivative strategy will help in reducing costs, as developing products results in lower incremental costs(Mohr, et al., 2009); hence in this paper a systematic change in a system is considered so that remote controlled machines will become fully autonomous robots. Hence, in literature review, we initially consider various types of robot systems from Robot autonomy Spectrum,networked robots, and cloud robots; and then various changes in hardware and software architectures are considered so that there is a smooth change from remote controlled machine to fully autonomous robots. Thus,this paper helps those developers of robots which supply robots for construction to develop various versions of robots, so that robots can be adopted in construction smoothly.

\section{Literature review}

There are many types of robots are there in Robot Autonomy Spectrum details of these robots are shown in the table 1 below.

Table 1: detail of types of robot from Robot Autonomy Spectrum

\begin{tabular}{|l|l|}
\hline Type & Detail \\
\hline Remote controlled machines & $\begin{array}{l}\text { Here the human operator will perform all sensing, planning, acting operations and machine } \\
\text { just follows the human, in this case human and machine is in closed vicinity. As primitives } \\
\text { are not supported by machine human operators are needed continuously. }\end{array}$ \\
\hline $\begin{array}{l}\text { Tele-operation controlled } \\
\text { machine }\end{array}$ & $\begin{array}{l}\text { Here the human operator acts as Master where controlled robot will be the slave. Hence the } \\
\text { main system has two main components, i.e. control module and Tele-manipulator or robot. A } \\
\text { Tele-operator cannot see directly what the robot is doing, hence sensors are provided to the } \\
\text { robot which gives information and then operators will provide instructions by using one } \\
\text { computer. Here communication link plays an important role between local and remote. }\end{array}$ \\
\hline $\begin{array}{l}\text { Semi-autonomous computer } \\
\text { control robot }\end{array}$ & $\begin{array}{l}\text { It is also called as supervisory control where instruction is given to the robot which it } \\
\text { performs safely on its own. There are two types of systems, i.e. continuous assistance system } \\
\text { and control trading. During continuous assistance system, human operator provides } \\
\text { instruction to the robot and monitors the task. Here the operator can relax when the robot is } \\
\text { working near the panel and help when something goes wrong, and shared control will help } \\
\text { the operator to avoid cognitive fatigues in case of repetitive and boring tasks. But high } \\
\text { communication bandwidth is needed. } \\
\text { In case of control trading with human just initiates an action for the robot and interacts to give } \\
\text { new commands if required. Here robot will perform all tasks autonomously. Thus a single } \\
\text { person can control many robots through a computer as a platform and human intervention } \\
\text { reduced. Here, robots can communicate with each other also just like a networked robot (D. } \\
\text { Song, et al. 2012), thus demand, for bandwidth reduces which results in reducing }\end{array}$ \\
\hline
\end{tabular}




\begin{tabular}{|l|l|}
\hline & communication problems. \\
\hline Fully autonomous robots & $\begin{array}{l}\text { Here the robot system will resolve choices on its own and has ability for complex decision } \\
\text { making, autonomous mission execution and planning, ability to self-adapt as a change in the } \\
\text { environment where it operates and able to understand system state and take action } \\
\text { accordingly. Here robot may act alone or in groups. }\end{array}$ \\
\hline
\end{tabular}

Source: (Sachin and Suman, 2016)

\section{Networked robots:}

(D. Song, et al. 2012) observes that networked robots are basically developed from tele-robots; however tele-operation has main problems about stability and time delay; multiple mobile robot systems concerns about coordination and planning of autonomous robots and sensors communicating over local networks. Hence networked robots improve from traditional master slave operation with integration of robot, human, agents, off board sensors, databases, etc. during supervisory control a local computer plays an active role in closing the feedback loop.Hence networked tele-robots fully evaluate into networked robots, i.e. an integration of robots, humans, computing power, off board sensing, and database over the internet. These networked robots are used in education, industry, commercial, health care, geology, environmental monitoring, entertainment and arts.Networked robot provides a new medium for people to interact with remote environment. The physical robot represents the remote person and also transmits multi model feedback to the person which is referred as tele-presence. Networked tele-robots provide universal access to the general public (who may not have knowledge of robots) with opportunities to understand, learn, and operate robots which were expensive scientific equipment limited to universities and large corporate laboratories. Networked tele-robot online remote laboratories improve distance learning by providing an interactive experience(D. Song, et al. 2012).

However, these networked robots have three main types of constraints due to resources; communication; and, due to learning along with information. (Guoqiang $\mathrm{Hu}$, et al, 2012).

\section{Cloud robots:}

Cloud robotic (CR) is introduced by James Kuffner in 2010 (Kuffner, 2010;D. Song, et al. 2012; Basit Qureshi, and Anis Koub^aa 2014; Busra KOKEN, and Gyula MESTER 2015) and now various companies like Google, Willow Garge and Gostai has many projects which give momentum to Cloud Robotics.

As traditional robots have constraints in terms of computational capability, memory and storage; CR helps them to overcome these challenges, and by using cloud cost effective robots can be produced. Robots can be classified as traditional robots and cloud enabled robotsCloud technology empowers robots also it allows them to network each other regardless of distance and cloud enabled robots are divided as standalone robot and networked robots. While Standalone robot can be benefited by cloud in terms of computational power, storage capacity and memory, whereas networked robots can make networks, share information through cloud and can perform collaborative works. With the help of cloud, robots can do many works like grasping, identifying objects, SLAM, monitoring, networking and other actuating works. (Busra KOKEN, and Gyula MESTER 2015).

(Gyula Mester, 2015) observes that CR combines robot technology with network and cloud computing infrastructure which connects, the amount of robot's sensors, portable devices and data centers. Robot web tools helps web developers, robotist person and students to build a robot web application and various routs are available for architecting a robot web application and Robot Operating System (ROS) is one of the popular robot middle wares, which provides libraries and tools to help software developers to create robot applications and also provide hardware abstraction, device drivers, libraries, visualizers, message passing, package management, etc., ROS is open source, BSD license (Gyula Mester, 2015).

Cloud robots has main benefits i.e. provide shared knowledge database which helps in organizing and unification of information regarding world is such a format which robot can understand; it helps in offloading of heavy computing to cloud which helps in developing light weight, cheap, and easily maintained hardware, improved battery life, lower requirement of software pushes and updates, invisible and hassle free upgrading of CPU; it provides skill and the behavior database, which helps in developing a reusable library of skills and behaviors which are required for the perceived task in complex situations, and data mining history of all cloud enabled robots (James Kuffner (2010).

Behavior and intelligence of robots can be improved through data mining into data sets along with data trajectories which sensors provide. For example, three indicators are there during human robot interaction include: synthesis of spoken language; gesture; and gaze. In case of cloud robotics mining of data found in various data sets along with knowledge base will help in robot intelligence, its interaction and behavior (Basit Qureshi, and Anis Koub^aa 2014).

Cloud has many limitations also, for example: it is not so helpful in robot's motion control as it depends on sensors and feedback; cloud based application may be slow or unavailable it robot depends on the cloud too much as any hitch in network leads to brainless robot; a task which has real time execution need on- 
board processing also. Some improvement is possible if the robot remembers conversations with the cloud which reduce brainless scenario; cloud aware android robot; and the app store for robots (Richard Voyles (2013) (José M. Reyes Álamo, et al, 2013) the cloud robots can use cloud computing along with mobile cloud. In case of the cloud robotics cloud helps in offloading heavy computation and storage needs of robot and robot will do only simple tasks; whereas during mobile cloud computing (MCC) mobile devices like smartphones along with tablets which gather data and used for simple calculation whereas cloud will perform tasks which are resource intensive. There are three possibilities of using robots, these include: mobile cloud robotics architecture; Android based mobile cloud robotics architecture; and hybrid. During mobile cloud robotics architecture a server is there between cloud and robot; robot will communicate with the server through Bluetooth, server will act as a gateway for command sending and receiving as it is online. The server also helps in some local computation. Here multi robots can be used through network formed by many interconnected robots; robots in the network also use the same architecture and uses Bluetooth for communication among themselves and the server, which improves scalability and elasticity.

In case of android device based robot communicates to Android device with Bluetooth or Wi-Fi; and server will be online and helps in commands sending and receiving along with doing some local computations. A network of robot can work similar to above architecture; however, as android support both Bluetooth and WiFi better connectivity is achieved, along with scalability and elasticity. Here robot handles sensors and android handles communication along with computation.

In case of hybrid type robot which has Android device performs communication among themselves and the server through Android device; whereas robots which does not have android device will use Bluetooth for the same task. The server will be responsible for communication with cloud and do some local computations. A network can also be developed (José M. Reyes Álamo, et al, 2013).

However, security and privacy of data; reliability of cloud solution; are the main concern when the cloud is used in cloud robotics (Report by KPMG, (2011).

\section{Changes needed for each type:}

Now we consider various changes which are needed in each type so that remote controlled machine will become fully autonomous robot, which is as shown in figure 1 below.

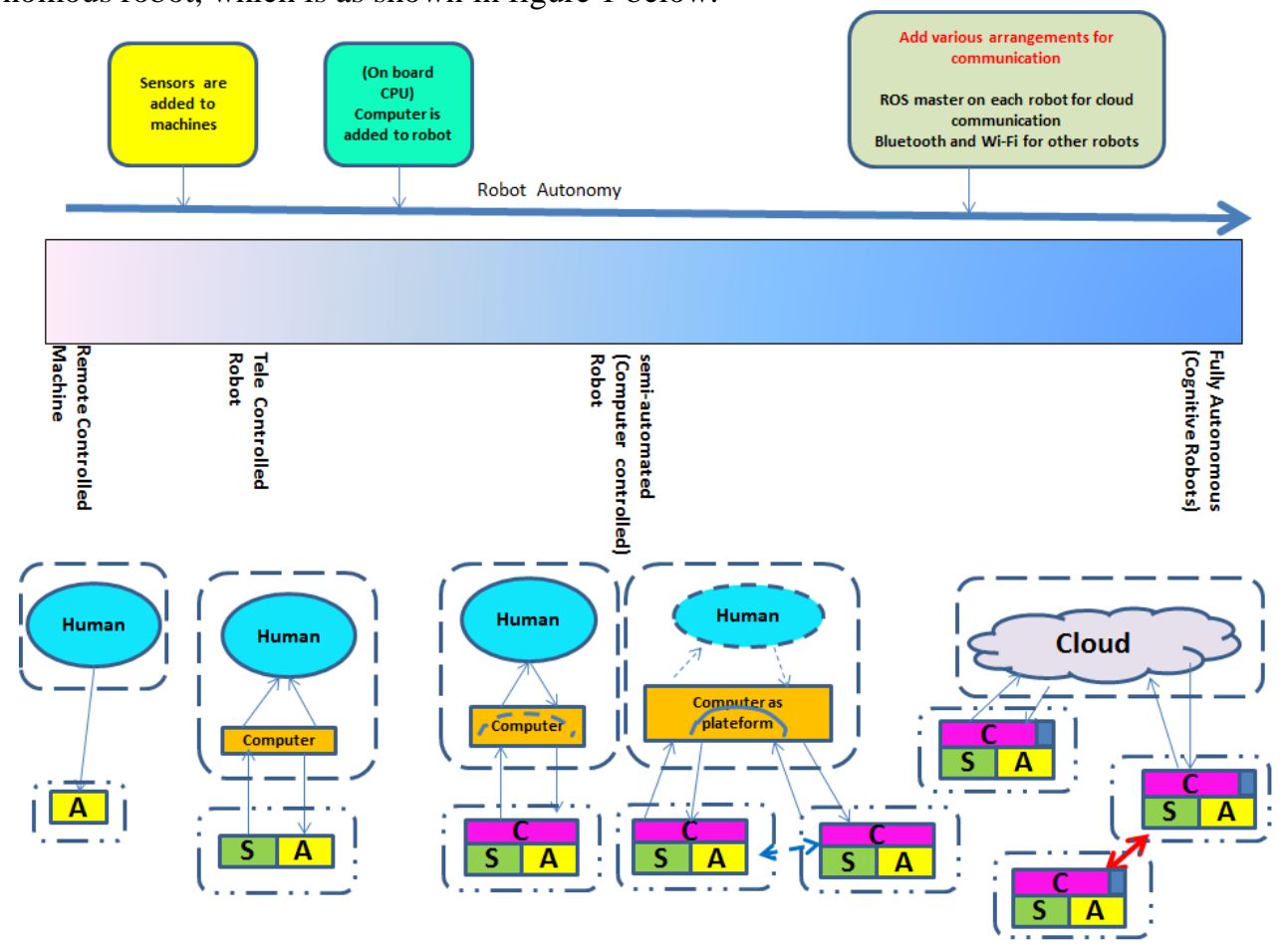

Figure: 1 Changes in the system to get fully autonomous robots

As shown in figure remote controlled machine system will have two main components, i.e. machine and controlling unit as human. Here a communication system is embedded in normal machines which help in controlling actuators of machines to perform task. The machine will be controlled by human operator from nearby; since the operator has to sense, plan and take decision by itself and the machine will just follow the instructions. 
When we want to convert remote machines to tele-operated robots, sensors must be added to machines and a computer is added tothe controlling unit so that robot can be controlled. Here robot will sense the environment by using sensors and provide information on computer. Which will help operators see information through display units and then operator will plan and provide instruction which is communicated to robot by computer. Hence operator may be located at a distance from the robot; however, delays in communication creates problems and sometime safety of the robot may be in danger as it follows the instructions. Here an operator is needed continuously and must provide instructions.

During semi-automated, i.e. computer controlled robot there must be some changes in robots, here we add an on board CPU, which will helps robot to perform reactive tasks; this CPU will also communicate with computer in controlling unit. Since the robot can perform reactive tasks, it is easier to safeguard itself (Robinr, 2000), which is important during the time delay. Thus, this system will help in continuous assistance system development. However single operator can control one robot only and a large communication bandwidth is required.

When a computer is used as a platform, it is possible to communicate with a large number of robots to perform a single complex tasks and human operator is just needed to give new instruction. In this case group of robots can also act as networked type and communicate among itself during tasks will reduce the communication width need. Also, this type has advantages as software is more distributed and robots can perform better. However,various constraints due to resources; communication; and, due to learning along with information are still needed to be solved.

Cloud robots help them to overcome these challenges; thus for achieving fully autonomous robots, we have to use the cloud for controlling the robots. Here cloud will help in getting smarter robots as cloud hardware is off the shelf which leads to affordable robot; it also helps as it reduces barriers to robot entry; and cloud provides scalable CPU, memory and storage (Damon Kohler et al, 2011). The Cloud can be used by a standalone robot or many robots in the network. All robots here also uses reactive architecture for lower layer, computer as middle layer along with ROS (Bingwei Liu, et, al, 2013) and other communication systems, including Android devices, Bluetooth, and Wi-Fi, etc., for communicating with cloud as well as among robots in a networked robotic system. Table 2 below show change in robot and controlling units for types of robots in Robot Autonomy Spectrum.

Table 2: Change in robot and controlling unit in types of robots in Robot Autonomy Spectrum

\begin{tabular}{|c|c|c|}
\hline Type of robot & Change in the robot & Change in controlling unit \\
\hline Remote controlled machines & $\begin{array}{l}\text { Ordinary machines } \\
\text { device }\end{array}$ & Human with remote for communication \\
\hline Tele-operated robots & Sensors + remote controlled machine & $\begin{array}{l}\text { Human uses for computer display and } \\
\text { commands }\end{array}$ \\
\hline $\begin{array}{l}\text { Semi-automated robot } \\
\text { (Continuous assistance system) }\end{array}$ & $\begin{array}{l}\text { Computer (on board CPU) }+ \text { Tele-operated } \\
\text { robot }=\text { Reactive robot }\end{array}$ & $\begin{array}{l}\text { Shared command by Human and computer; } \\
\text { however robot can perform task } \\
\text { independently and then wait for instruction } \\
\text { if changes are there.But here single robot is } \\
\text { used. }\end{array}$ \\
\hline $\begin{array}{l}\text { Semi-automated robot } \\
\text { (Control trading system) }\end{array}$ & $\begin{array}{l}\text { Computer }(\text { on board CPU) }+ \text { Tele-operated } \\
\text { robot }=\text { Reactive robot }\end{array}$ & $\begin{array}{l}\text { Here computer platform is used for } \\
\text { controlling many robots which many also } \\
\text { communicate among them as networked } \\
\text { robots. Human is used if changes are } \\
\text { needed. }\end{array}$ \\
\hline Fully autonomous robots & $\begin{array}{l}\text { Reactive robots }+ \text { ROS }+ \text { Android devices } \\
\text { for communication with cloud; and } \\
\text { Bluetooth and Wi-Fi for communication } \\
\text { with other robots }\end{array}$ & $\begin{array}{l}\text { Cloud is used for helping standalone robot } \\
\text { or networked robots; robots normally } \\
\text { perform activity on its own, however, can } \\
\text { take help of cloud for heavy computation } \\
\text { and storage needs, along with libraries for } \\
\text { behavior. }\end{array}$ \\
\hline
\end{tabular}

\section{Conclusion}

Fully autonomous robots can be developed from ordinary machines by considering changes which lead to various platforms or next generation products i.e. robots. Here development must be there in both components, i.e. robots and controlling unit. As we have seen that during controlling unit control is shifted from human to cloud which need less assistance for humans. Similarly,the machine will have the addition of sensors, computers, i.e. on-board CPU along with ROS, android, Bluetooth and Wi-Fi for communication. Also the use of cloud will help in many ways, i.e. in case of standalone robot it helps in computational power, storage capacity, memory; similarly in case of networked robots, it helps to share information and performs collaborative tasks. Cloud robot also has shared knowledge database which helps the robot to understand the world in a better way; cloud also helps in developing reusable libraries of skills and behaviors which is very useful during complex tasks in construction. Also cloud helps robot to become more intelligent and improve its behavior, which leads to full autonomous robots. Thus the developer of robot for construction industry can develop four versions. 


\section{References}

[1]. Jain, Sachinand Jain, Suman. (2016). Robot Autonomy Spectrum for Proper Adoption of ROBOTS in Construction Industry. GEInternational Journal of Engineering Research. Vol. 4, No. 4, pp. 10-26

[2]. Mohr,J., Sengupta,S., and Slater,S. (2009)Marketing Of High Technology Products And Innovations. India, Dorling Kindersley (India) Pvt Ltd Licenses Of Pearson Education in South Asia, 2009, (2nd Ed)

[3]. Busra KOKEN, and Gyula MESTER (2015), "The evolution of cloud robotics: a survey", ACTA TEHNICA CORVINIENSIS Bulletin of Engineering, Tome VIII [2015] Fascicule 2 [April - June], ISSN: 2067 - 3809

[4]. Guoqiang Hu, Wee Peng Tay, and Yonggang Wen, Nanyang Technological University (2012), "Cloud Robotics:Architecture, Challenges and Applications", IEEE Network

[5]. Basit Qureshi, and Anis Koub^aa (2014), "Five Traits of Performance Enhancement using Cloud Robotics: A Survey", Procedia Computer Science 00 (2014) 000-000

[6]. Gyula Mester (2015), “Cloud Robotics Model”, Interdisciplinary Description of Complex Systems 13(1), 1-8, 2015

[7]. José M. Reyes Álamo, Benito Mendoza and Aparicio Carranza (2013), "towards an architecture for mobile cloud robotics", IHART - Volume 31 (2013), pp 391-398

[8]. Damon Kohler, Ryan Hickman, Ken Conley, Brian Gerkey (2011), “Cloud Robotics”, report by Google

[9]. Report byKPMG, (2011), "The CloudChanging the Business Ecosystem: Setting the Context", KPMG international cooperative, KPMG networks, pdf is created on 8/2/2011

[10]. James Kuffner (2010), "Cloud enabled Humanoid Robots", the Robotics Institute, Carnegie Mellon Univercity, pdf is created on 22/12/2010

[11]. Richard Voyles (2013), "Robotics as a "Singularity": The Case for Cloud Robotics and Real-Time Big Data",Program Director, National Science Foundation, Computer \& Information Science and Engineering Directorate

[12]. Robinr Murphy (2000), "Introduction to AI Robotics", A Bradford Book, The MIT Press, Cambridge, Massachusetts, London, England

[13]. Bingwei Liu, Yu Chen, Erik Blasch, Khanh Pham, Dan Shen, Genshe Chen (2013), “A Holistic Cloud-Enabled Robotics System for Real-Time Video Tracking Application", pdf is ceated on 27/3/2013 